\title{
Spin Droplet Formation in Quantum Dots
}

M. C. Rogge, E. Räsänen, and R. J. Haug

Citation: AIP Conference Proceedings 1399, 301 (2011);

View online: https://doi.org/10.1063/1.3666373

View Table of Contents: http://aip.scitation.org/toc/apc/1399/1

Published by the American Institute of Physics 


\title{
Spin Droplet Formation in Quantum Dots
}

\author{
M. C. Rogge*, E. Räsänen ${ }^{\dagger}$ and R. J. Haug* \\ ${ }^{*}$ Institut für Festkörperphysik, Leibniz Universität Hannover, Appelstrasse 2, 30167 Hannover, Germany \\ ${ }^{\dagger}$ Nanoscience Center, Department of Physics, University of Jyväskylä, FI-40014 Jyväskylä, Finland
}

\begin{abstract}
A lateral quantum dot with approx. 50 electrons is analyzed in high perpendicular magnetic fields. In a regime with two Landau levels (LL) in the quantum dot information about the spin structure is gained by investigating the position of Coulomb blockade peaks. A typical zig-zag pattern is observed which is roughly explained with the so called Constant Interaction model. However, only states from LL0 can be approximated with this model, while for LL1 more complex electron-electron interactions must be included. This is done with spin density functional theory calculations. As a result, full spin polarization is found for LL1, the so called spin droplet.
\end{abstract}

Keywords: Quantum dots, Coulomb blockade, single-electron tunneling

PACS: 73.21.La, 73.23.Hk, 73.63.Kv

The properties of many electron quantum dots still provide many open questions. Especially in perpendicular magnetic fields research never came out of focus and many interesting results have been gained so far. The most significant features like the formation of Landau levels (LL) could be explained using a single particle approach, the so called Constant Interaction model (CI-model) [1, 2]. Then self consistent calculations were applied to model electron densities [3]. Further investigations concentrated on spin properties. Many spin related effects were found, e.g. spin blockade, Kondo physics, spin polarizations etc. [4, 5, 6, 7, 8].

Here we present a combined experimental and theoretical work on the spin configuration of a lateral quantum dot with approx. 50 electrons in high perpendicular magnetic field. The quantum dot is made with local anodic oxidation on a GaAs/AlGaAs heterostructure. It is connected to a Source- and a Drain-lead to allow for transport measurements. A plunger gate is added to tune the potential and the number of electrons. Further details about the sample can be found in [9].

The dot is set in the Coulomb blockade regime with approx. 50 electrons. Several Coulomb peaks are investigated in a perpendicular magnetic field $B$. Figure 1 a) shows the peak positions of two successive Coulomb peaks as a function of $B$ and energy $E$. Both peaks show a pronounced zig-zag pattern having sections with increasing energy as a function of $B$ and sections with decreasing energy. These sections belong to different electronic states that cross as a function of $B$. At each such crossing the energetically favored (and therefore occupied) state is changed. In the investigated field range states with decreasing energy belong to LL0 while states with increasing energy belong to LL1.

These zig-zag patterns are in principle explained with the CI-model, that describes the electronic many body
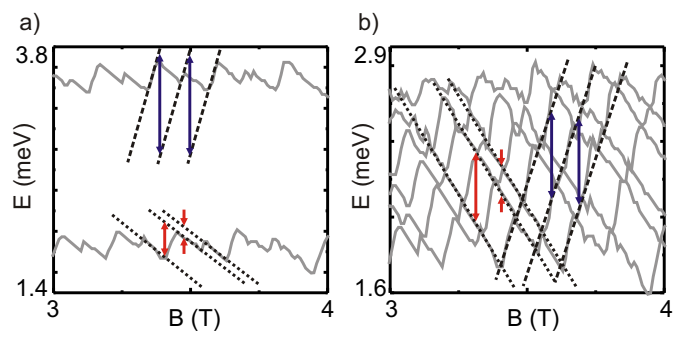

FIGURE 1. a) energetic peak positions of two successive Coulomb peaks as a function of $B$. A zig-zag pattern is formed by states from LLO and LL1. Energetic distances of adjacent states in LL0 (red arrows) and LL1 (blue arrows) can be measured for each Coulomb peak. b) several Coulomb peaks can be shifted together to measure these distances averaged over several electron numbers.

states of the quantum dot with a single particle excitation spectrum (commonly the Fock-Darwin spectrum [10, 11] with spin) plus a constant Coulomb interaction due to the electronic charge. This is of course a simplified approach that fails when the measurements are investigated in detail. We are especially interested in the electronic distances of adjacent states within a certain Landau level. According to the CI-model, a bimodality should be observed independent of the Landau level. These distances can be measured on a single Coulomb peak like shown in Fig. 1 a). At the lower Coulomb peak, the red arrows mark the distances between adjacent states from LL0, while at the upper peak distances within LL1 are marked with blue arrows. A more accurate approach is to average the distances over several Coulomb peaks by shifting these peaks together. This is done in Fig. $1 \mathrm{~b}$ ). Six Coulomb peaks are shifted together such that a pattern of crossing lines appears and electronic states can be 

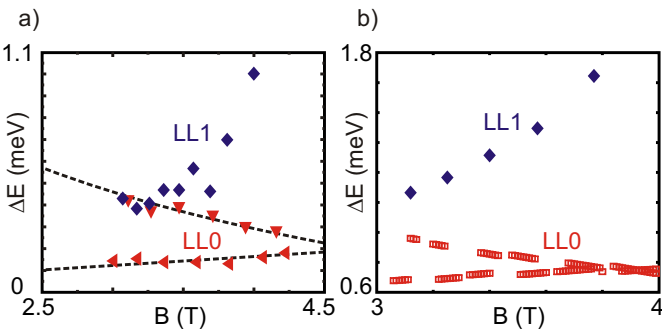

FIGURE 2. a) The energetic distances of adjacent states show two branches for LL0 and one branch for LL1. The CImodel can only explain the branches of LL0 (dashed lines). b) The measurement is resembled qualitatively with SDFTcalculations.

followed over several electron numbers. Again, energetic distances of adjacent states can be measured for LLO (red arrows) and LL1 (blue arrows).

The results of the latter method are presented in Fig. 2 a). The energetic distances $\Delta E$ within each Landau level are plotted as a function of $B$. As expected from the CImodel, LL0 shows two branches reflecting the before mentioned bimodality. And indeed these branches can be fit assuming a Fock-Darwin spectrum with spin. The two branches are explained by the two electronic spins. Either a new orbital is filled with spin up or a half filled orbital is filled with spin down. According to the CImodel, this should also account for LL1. However, there is only one branch at higher energy with positive slope. The CI-model is therefore insufficient.

In order to solve this problem, we applied spin density functional theory (SDFT) [12]. The $N$-electron Hamiltonian is considered as

$$
\begin{aligned}
H & =\frac{1}{2 m^{*}} \sum_{i=1}^{N}\left[\mathbf{p}_{i}+e \mathbf{A}\left(\mathbf{r}_{i}\right)\right]^{2}+\sum_{i<j}^{N} \frac{e^{2}}{4 \pi \varepsilon_{0} \varepsilon\left|\mathbf{r}_{i}-\mathbf{r}_{j}\right|} \\
& +\sum_{i=1}^{N}\left[V_{\operatorname{ext}}\left(\mathbf{r}_{i}\right)+E_{z, i}\right],
\end{aligned}
$$

with $\mathbf{A}$ the external vector potential of the magnetic field $\mathbf{B}=B \hat{z}$, the external harmonic 2D-potential $V_{\text {ext }}(r)=$ $m^{*} \omega_{0}^{2} r^{2} / 2$ with $\hbar \omega_{0}=4 \mathrm{meV}$, and the Zeeman energy $E_{z}=g^{*} \mu_{B} B s_{z}$. We use the GaAs material parameters: $m^{*}=0.067$ and $\varepsilon^{*}=12.4$. A gyromagnetic ratio $g^{*}=$ -0.30 is chosen.

The result of these SDFT-calculations is presented in Fig. 2 b). In qualitative agreement to the measurements, indeed three branches are found. There are two branches for LLO and one branch at higher energy and with a positive slope for LL1. Thus the measurement indeed shows a many body effect due to electron-electron interaction beyond a constant Coulomb repulsion. The reason for this many-body effect is the spin configuration of the two Landau levels. As explained before, the two

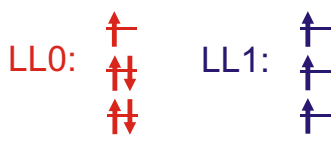

FIGURE 3. The results are explained with two different spin configurations in both Landau levels. While LL0 is filled unpolarized with spin up and spin down, there is a spin polarization in LL1, the so called spin droplet.

branches of LLO are due to the two electronic spins, as LLO is filled regularly with spin up and spin down, according to the CI-model. this result is confirmed with the SDFT-calculations. In contrast to the CI-model, the SDFT-calculations show, that LL1 is completely spin polarized with spin up as schematically shown in Fig. 3. This spin polarization of the highest Landau level was predicted theoretically for quantum dots with more than 30 electrons and measured indirectly $[13,14,15]$. Here a direct confirmation of this so called spin droplet is achieved.

In conclusion we have investigated the peak positions of Coulomb peaks of a 50 electron lateral quantum dot in high perpendicular magnetic field. We have found a zigzag pattern that can not be explained with a single particle approach like the CI-model. However, with SDFTcalculations we were able to explain our results qualitatively. As a result we found a direct confirmation for the spin polarization of the highest Landau level, the so called spin droplet.

This work has been supported by BMBF via nanoQUIT, by QUEST, and by the Academy of Finland.

\section{REFERENCES}

1. C. W. J. Beenakker, Phys. Rev. B 44, 1646 (1991).

2. U. Meirav and E. B. Foxman, Semicond. Sci. Technol.11, 255 (1996).

3. P. L. McEuen et al., Phys. Rev. B 45, 11419 (1992).

4. M. Stopa et al., Phys. Rev. Lett. 91, 046601 (2003).

5. M. Keller et al., Phys. Rev. B 64, 033302 (2001).

6. M. Ciorga et al., Phys. Rev. B 61, R16315 (2000).

7. M. C. Rogge, C. Fühner, and R. J. Haug, Phys. Rev. Lett. 97, 176801 (2006).

8. A. Wensauer, M. Korkusinski, P. Hawrylak, Phys. Rev. B 67, 035325 (2003).

9. M. C. Rogge et al., Appl. Phys. Lett. 85, 606 (2004).

10. V. Fock, Z. Phys. 47, 446 (1928);

11. C. G. Darwin, Math. Proc. Cambridge Philos. Soc. 27, 86 (1930).

12. For a review see, e.g., R. M. Dreizler and E. K. U. Gross, Density functional theory (Springer, Berlin, 1990).

13. E. Räsänen et al., Phys. Rev. B 77, 041302(R) (2008).

14. A. Harju, H. Saarikoski, and E. Räsänen, Phys. Rev. Lett. 96, 126805 (2006).

15. H. Saarikoski et al., Phys. Rev. B 78, 195321 (2008). 